\title{
CRYOGENIC DETECTORS EXPLORING NEW PHENOMENA IN PHYSICS AND ASTROPHYSICS
}

\author{
| Klaus Pretzl - Laboratory for High Energy Physics at the University of Bern, Switzerland - DOI: https://doi.org/10.1051/epn/2021303 \\ The potential to measure small energy transfers with very high energy resolutions \\ motivated the development of cryogenic detectors to search for dark matter \\ in the universe, the neutrino mass, neutrinoless double beta decay, and new phenomena \\ in astrophysics. Other fields like material and life sciences also benefited from \\ these developments.
}

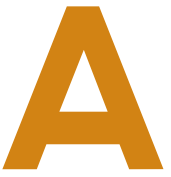

typical cryogenic calorimeter consists of an absorber, a thermometer which measures the temperature increase due to the en-

ergy transfer of a particle in the absorber, and a thermal bath which restores the temperature of the absorber to its original base value. In contrast to other devices, cryogenic calorimeters measure the total deposited energy in form of ionization and heat (phonons). They can be made from different materials including superconductors, a feature which turns out to be very useful for many applications. Most detectors use superconducting materials since there are very low energy quanta involved. It only takes energies of the order of a meV to break a Cooper pair in a superconductor, as compared to a few $\mathrm{eV}$ which are needed to create an electron hole pair in a solid state device. Due to large quantum statistics, energy resolutions of typically a few eV for small energy transfers of a few $\mathrm{keV}$ can be achieved with most cryogenic detectors. This is more than an order of magnitude better than what can be reached with solid state devices. A drawback is that they have to operate at $\mathrm{mK}$ temperatures requiring complex refrigeration systems, and that they have limited rate capabilities $(1 \mathrm{~Hz}-1 \mathrm{kHz})$.

Cryogenic detectors differ in the way they are converting the excitation energy in the calorimeter into a measurable signal. There are devices using phonon sensors, quasiparticle detection or magnetic thermometers. Excitations in the absorber produce electrons, photons (photoelectrons) and phonons, which degrade in time via electron- phonon and phonon-phonon interactions until the system reaches a thermal equilibrium. Calorimeters operating in the equilibrium mode deliver the best energy resolutions, but are intrinsically slow, providing second to millisecond signals. In some applications they are also used in a non-equilibrium mode, which makes them faster ( microsecond signals), but with inferior energy resolutions.

\section{Phonon sensors}

Semiconducting thermistors and superconducting transition edge sensors (TES) are used as resistive thermometers. They measure the change of a resistor as result of a temperature change in an absorber. Due to the long thermalisation and recovery time of the system they have slow response signals which limits their counting rate capability to a few $\mathrm{Hz}$. They also have to deal with Joule heating which results from the power dissipation of the thermometer read out current into the system. However, for sensors connected to a voltage biased electrothermal feedback system the effect of Joule heating can be largely reduced. This feedback also stabilizes the operating temperature of the system in a self-calibrating mode. Other phonon sensors are magnetic thermometers with an inductive readout. They do not dissipate power into the system.

A thermistor is a heavily doped semiconductor below the metal insulator transition. Its conductivity results from a phonon driven electron-hopping mechanism between impurity sites. Good uniformity of doping concentrations has been achieved either with ion implantations or with neutron transmutation doping (NTD). With thermistors very high energy resolutions can be achieved. In addition, they are easy to handle and commercially available. This makes them very attractive for many applications.

A TES sensor consists of a superconducting strip or film attached to an absorber. It operates at a temperature 
in the narrow transition region between the superconducting and the normal phase. The very steep resistance change versus temperature in the transition region provides a high sensitivity to very small energy transfers in the absorber. Superconducting TES sensors can be attached to absorbers made from different materials. But they can also be used as absorber and sensor at the same time. Superconducting TES detectors are also sensitive to non- thermal phonons (ballistic phonons) with energies larger than the binding energy of Cooper pairs. These phonons produce quasiparticles before they thermalize. Since this process is much faster than thermalization, it enhances the counting rate capability considerably, up to several $\mathrm{kHz}$. The application of the abovementioned auto-biasing electro-thermal feedback system turns out to be very advantageous for the operation of large pixel detectors or calorimeter arrays. Superconducting TES sensors belong to the most advanced cryogenic detectors with many applications in numerous fields of research.

Magnetic sensors make use of the temperature dependence of magnetic properties of metallic materials. Sensitive magnetic calorimeters (so called metallic magnetic calorimeters, MMC) are made from paramagnetic strips in thermal contact to an absorber. They are placed in an external magnetic field. A temperature change in the absorber leads to a change in the magnetization of the sensor, which can be measured with a SQUID (superconducting quantum interference device) magnetometer.

\section{Quasiparticle detection}

External particle interactions in a superconductor lead to the breaking of Cooper pairs and quasi-particle production. The energy loss of the particle is proportional to the number of quasi-particles produced. It can be measured with a superconducting tunnel junction (STJ), more widely known as Josephson junction. When biasing the STJ at a suitable voltage, the tunnelling current is proportional to the excess number of quasi-particles produced. A typical STJ consists of two thin superconducting films separated by a very thin tunnel barrier, which is usually made from the oxyde of one of the superconductors. In order to keep the tunnelling time shorter than the recombination time of the quasi-particles, the overlap region of the films $(100 \times 100$ micrometer squared) and the resistance in the junction have to be $\bullet \bullet$

If the dark matter in the universe consists of weakly interacting particles (WIMPs), it can potentially be detected by measuring the nuclear recoil energy in elastic WIMP-nucleus scatterings. $\nabla$ म

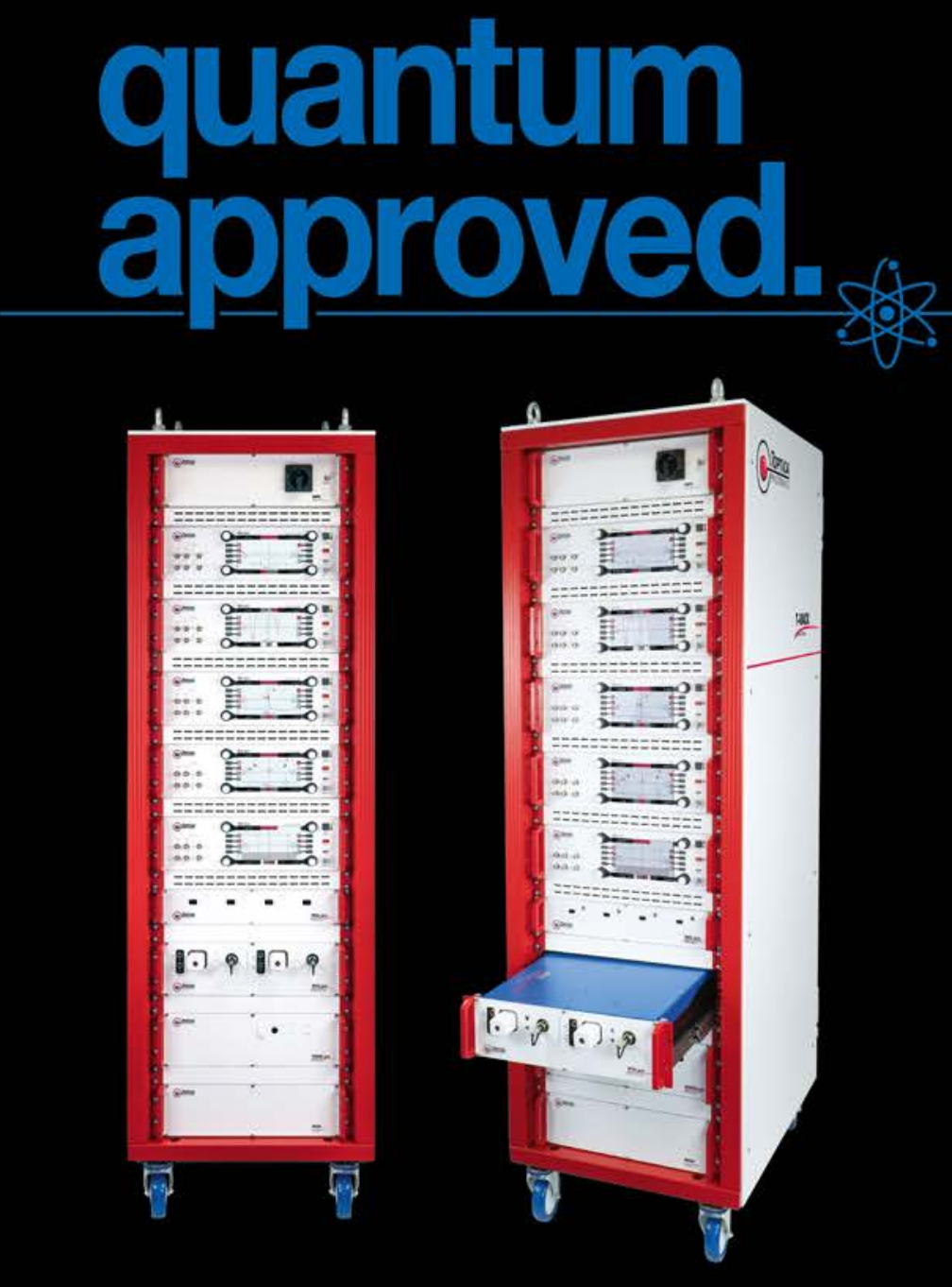

\section{Laser Rack Systems}

Quantum Technology meets Industry Standards

Our lasers do not need an optical table!

The T-RACK is the perfect home for

TOPTICA's high-end tunable diode lasers and frequency combs in a modular 19" form factor. Pick yours!

- Tunable Diode Laser Systems

- Frequency Comb Systems

- Wavelength Meters

- Locking Electronics

- $330 . .1770 \mathrm{~nm}$ 
V FIG. 1:

A module of the cryogenic dark matter detector of the

CRESST experiment in the Gran Sasso

Underground

Laboratory in Italy

(Photo credit: T.

Dettlaff, Max-

Planck-Institut für

Physik, München).

(c) T.Dettlaff, Max

Planck Institut für

Physik in München.
- kept very small. However, for very thin films the quantum efficiencies for X-ray detection are very low. This can be improved by choosing a larger size superconducting absorber as substrate to the STJ. Quasiparticles will be trapped in the junction when the absorber material has a higher energy gap with respect to the junction. STJ devices are frequently used as multi-pixel antennas in astrophysical observations.

Another attractive quasiparticle sensor is the microwave kinetic inductance detector (MKID). It consists of a thin superconducting film which is part of a transmission line resonator. MKID operates in a non-equilibrium mode. Quasi-particles produced by Cooper pair breaking of incident photons will change the inductive surface impedance of the superconductor and consequently the transmission phase of the resonator. The change of the transmission phase is proportional to the number of produced quasi-particles and thus to the energy of the incident photons. MKID allow simple multiplexing by coupling an array of many resonators with slightly different resonance frequencies to a common transmission

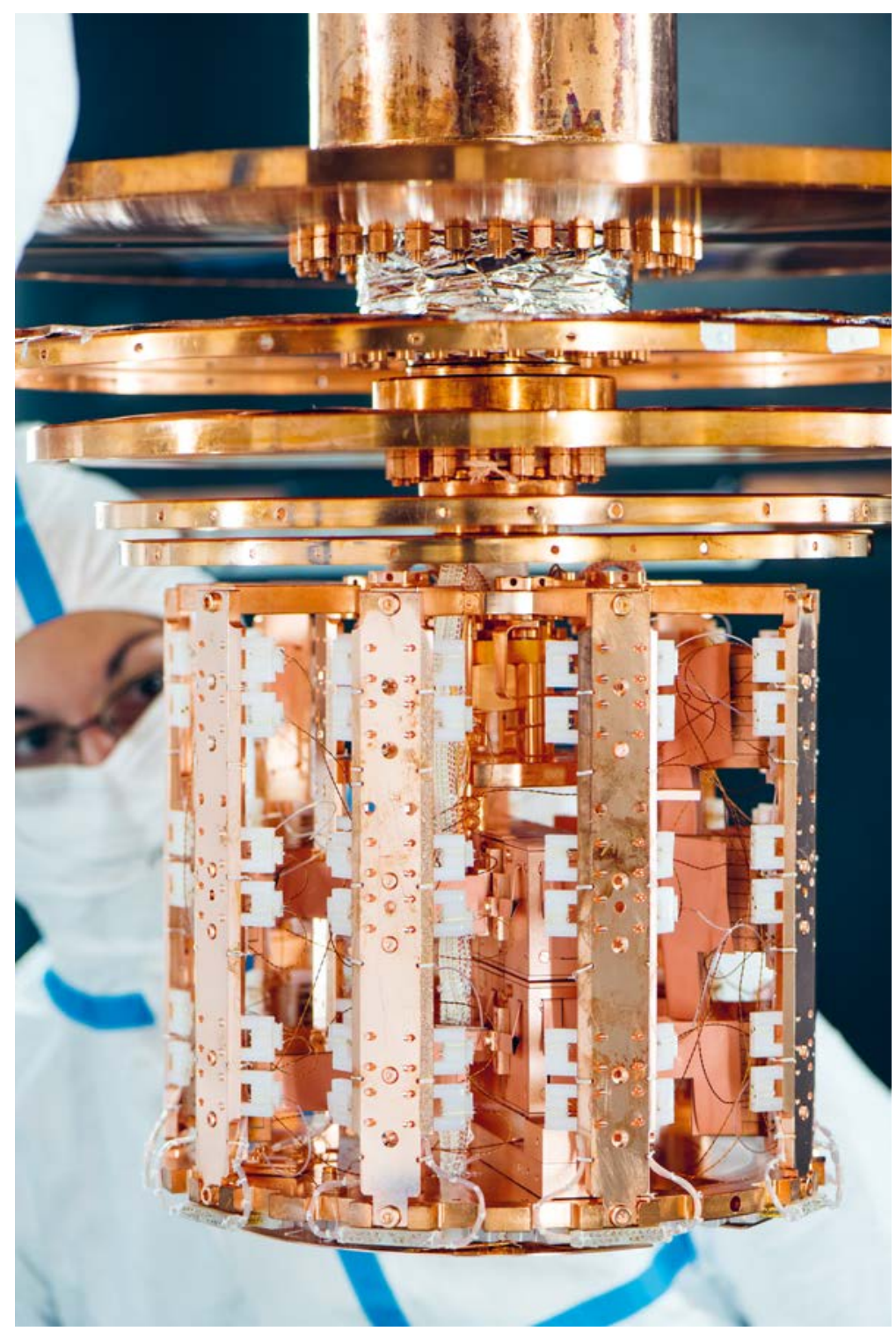

line. As a result, only one amplifier is required to treat the signals from a large number of detectors. This feature allows for a simple multiplexing of large readout systems. MKID based detectors find many applications in multipixel X-ray and single photon cameras.

An early development of cryogenic detectors have been superheated superconducting granules (SSG). A SSG detector consists of millions of small size grains with a typical diameter of 30 micrometer diluted in a dielectric material and embedded in an external magnetic field. The grains are made from superconducting materials of type- $1(\mathrm{Sn}, \mathrm{Al}, \mathrm{Pb}, \ldots)$ and can be produced industrially. SSG acts as absorber and detector at the same time. An energy transfer in a single grain can lead to the transition of the grain from a metastable superconducting to a normal conducting state due to the disappearance of the Ochsenfeld-Meissner effect. Transitions of a single grain in a very large sample are detected by a conventional pick-up coil or a SQUID magnetometer. Primarily used as a threshold detector, it has so far found limited applications. Nevertheless, it was successfully employed in a neutron scattering experiment and in an early dark matter search.

\section{Applications}

If the dark matter in the universe consists of weakly interacting particles (WIMPs ), it can potentially be detected by measuring the nuclear recoil energy in elastic WIMP-nucleus scatterings. Dark matter searches are in an advanced stage requiring more and more massive detectors to increase their sensitivity. Cryogenic calorimeters have the potential to detect very small recoil energies and to use a very large variety of detector materials. Large recoil energies and high sensitivities can be achieved if the atomic mass to the detector is matched to the WIMP mass. Massive cryogenic calorimeters of several $10 \mathrm{~kg}$ are already in operation. They complement the even more massive (ton-range) liquid Xenon and liquid Argon calorimeters, which are already employed in deep underground dark matter experiments.

The discovery of neutrino oscillations by the Kamiokande experiment in Japan suggests that neutrinos are massive. Several experiments now focus on the questions: What is the absolute mass of the neutrinos, and are the neutrinos and their antiparticles different or identical particles (i.e. Dirac or Majorana type particles). An answer to the latter question can be searched for in the nuclear double beta decay, a process, which was suggested in 1935 by Maria Goeppert Mayer. This transition can occur in two ways, where either two electrons and two antineutrinos or only two electrons end up in the final state. An observation of a neutrinoless final state would imply that the neutrino cannot be distinguished from its antiparticle. In search for this transition, the double beta decay in Tellurium-130 was chosen because of its 
high transition energy and isotopic abundance. Massive (1 ton) cryogenic detectors using Tellurium absorber crystals and Germanium NTD thermistors are under construction in order to surpass the presently reached sensitivities for this decay mode.

Direct neutrino mass experiments aim to measure the end point of the energy spectrum of electrons in beta-active nuclei with high precision. Spectroscopic measurements of the electrons often have to deal with final state interactions in the radioactive source. Cryogenic detectors circumvent these problems by measuring the total energy of the electrons including the final state interactions. The Rhenium- 187 beta decay is a good candidate for this investigation since it has a low endpoint energy $(2.6 \mathrm{keV})$ and a large isotopic abundance. Rhenium is also a superconductor and well suited as a cryogenic detector. Also the electron capture decay of Holmium-163 is being considered for such an investigation.

Astrophysics is a rapidly growing field requiring instruments with broadband capability, high spectral resolving power, efficient photon counting and large area imaging properties. Large pixel arrays of cryogenic detectors are already in use in many astronomical observatories. Because of their wide spectral sensitivity, they are more and more replacing conventional dispersive spectrometers. Cryogenic pixel arrays have also been employed to investigate the polarization of the cosmic microwave background radiation(CMB). These polarization measurements could provide revealing insight into the inflationary scenario of the early universe, signalling the effect of primordial gravity waves.

Present developments of cryogenic detectors are concentrating upon low mass dark matter particle (below $1 \mathrm{GeV} / \mathrm{c} 2$ ) and coherent neutrino scattering searches as well as upon the technical realization of large pixel arrays for astrophysics observations and the multiplexing of large electronic readout systems. A more detailed description of cryogenic detectors and their applications can be found in Refs. 1 and 2, and in the references therein.

\section{About the author}

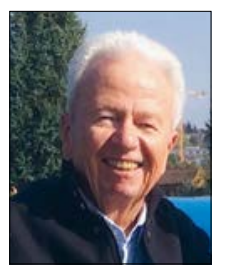

Klaus Pretzl is Professor emeritus of Experimental Physics at the Laboratory for High Energy Physics at the University of Bern. He has also been actively involved in the development of cryogenic detectors.

\section{References}

[1] Ch. Enss (ed.), Cryogenic Particle Detection, Topics in Applied Physics 99, Springer (2005)

[2] K.Pretzl, Cryogenic Detectors in Particle Physics Reference Library (eds. Ch. Fabjan and H. Schopper) 2, page 871 (2020) Open Access Springer

\section{n

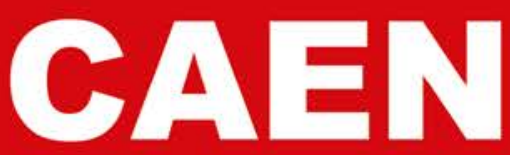 \\ Electronic Instrumentation}

\section{NIM FAMILIES}

\section{Desktop and Double-width NIM modules}

\section{NEW DT1081A - N1081A Four-Fold Programmable Logic Unit}
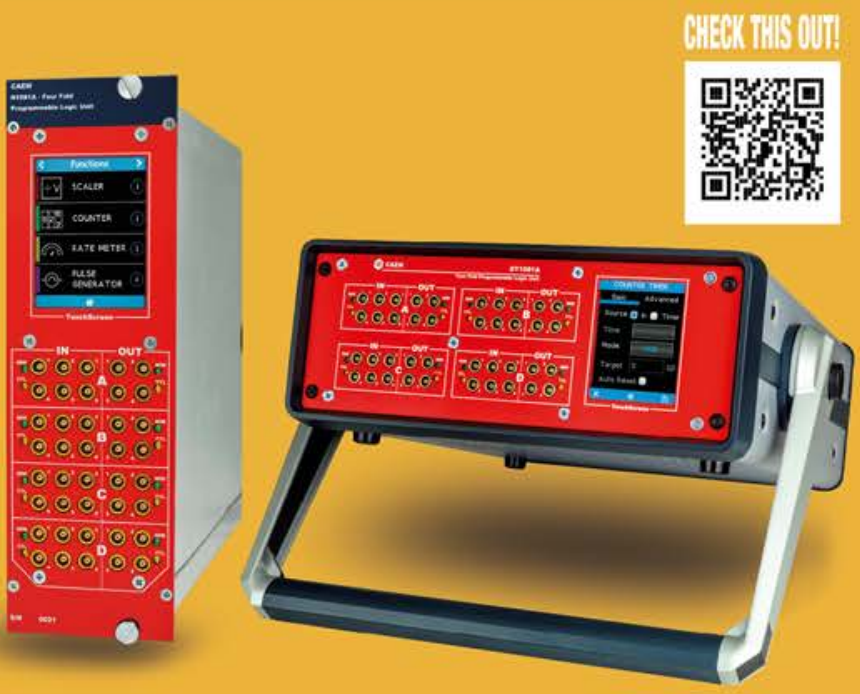

Save your time, enhance your success!

The DT1081A and N1081A are a laboratory tool that incorporates in a single modiule the most common functionalities that you need to implement the logic oapabilities of your experiment.
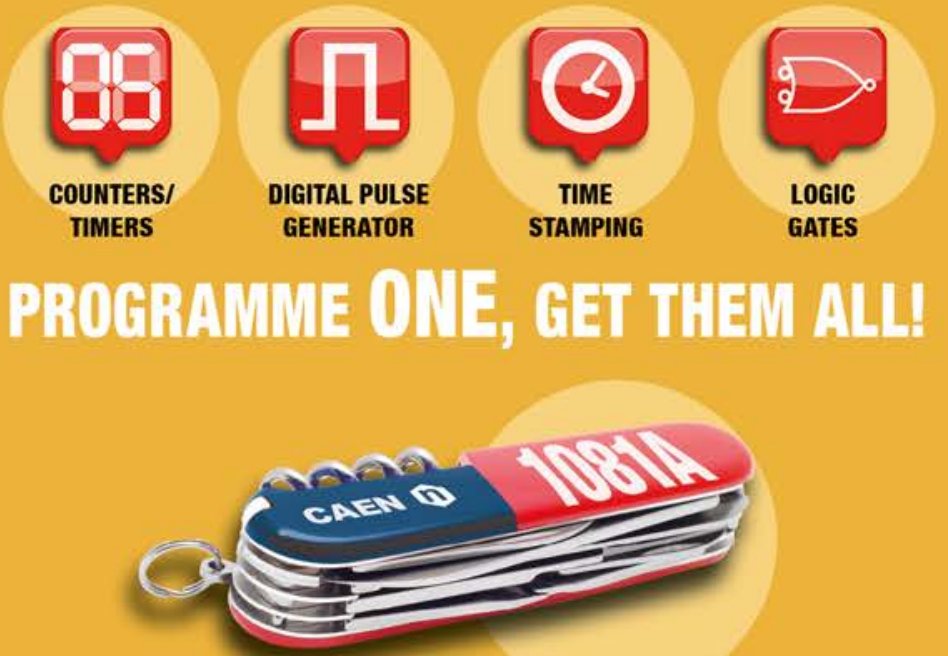

A new CAEN Tool in your Laboratory! 